\title{
Encoding of the unconditioned stimulus in Pavlovian conditioning
}

\author{
RUTH M. COLWILL \\ Brown University, Providence, Rhode Island \\ and \\ DAPHNE K. MOTZKIN \\ University of Pennsylvania, Philadelphia, Pennsylvania
}

\begin{abstract}
Three experiments, using rats, demonstrated the encoding of a food unconditioned stimulus (US) in a simple Pavlovian conditioning paradigm. In all three studies, one stimulus was used to signal the delivery of pellets and a different stimulus was used to signal the delivery of sucrose. In Experiment 1 , postconditioning devaluation of one of the food USs selectively reduced the frequency of conditioned magazine-directed behavior during the stimulus trained with that US. In Experiment 2, transfer of the stimuli to instrumental responses resulted in selective depression of the response trained with a different outcome. In Experiment 3, acquisition of stimulus-outcome learning was impaired by unsignaled intertrial presentations of the same outcome but not of a different outcome. These results indicate that a detailed representation of the outcome is encoded in the normal course of Pavlovian conditioning.
\end{abstract}

A major goal in the study of Pavlovian conditioning and instrumental learning has been the identification of the elements that become associated. The purpose of the present series of experiments was to apply the techniques that have been developed for an analysis of instrumental learning to the study of Pavlovian conditioning. Three studies are described, each of which attempts to provide the kind of evidence for a detailed encoding of the outcome in Pavlovian conditioning that is analogous to that reported by Colwill and Rescorla (1985b, 1986, 1988) for instrumental learning.

Colwill and Rescorla have supplied three kinds of support from their studies with rats for an encoding of the outcome in instrumental learning; in two cases, they used postlearning manipulations (Colwill \& Rescorla, 1985b, 1988), and in the third case, they manipulated the conditions that produce learning (Colwill \& Rescorla, 1986). One postlearning manipulation involved probing the sensitivity of instrumental responses to the current value of the outcomes used to establish them (Colwill \& Rescorla, 1985b). Performance of a response whose outcome had been reduced in value was less likely than that of a response whose outcome was still attractive. This specificity suggests that associations had formed be-

These experiments were supported by National Science Foundation Grants BNS 83-08176, to R. A. Rescorla, and BNS 89-15342, to R.M.C.; by a Brown University grant, BRSG 5-27469, to R.M.C.; and by an award from the Nassau Fund of the University of Pennsylvania to D.K.M. We thank R. A. Rescorla for helpful comments on a previous version of this manuscript. Correspondence concerning this article should be addressed to R. M. Colwill, Department of Psychology, Brown University, Box 1853, Providence, RI 02912. tween representations of each of the outcomes and the particular responses that had produced them. The other postlearning manipulation used by Colwill and Rescorla (1988) involved an assessment of the effect of discriminative stimuli trained with different outcomes on instrumental responses trained with those outcomes. Performance of a response was increased by presentations of a stimulus trained with the same outcome, but was unaffected by presentations of a stimulus trained with a different outcome. The dependence of this interaction between stimuli and responses on outcome identity provides additional support for a detailed encoding of the outcome.

The third line of evidence for encoding of outcomes comes from a study of the conditions that produce learning. Colwill and Rescorla (1986) examined the effect of degrading the response-outcome contingency on instrumental performance by noncontingent presentations of the outcome. They found that performance of a response that earned the same outcome as that delivered noncontingently was significantly reduced compared with a response that earned a different outcome. Two additional observations support the view that this performance decrement was mediated by a reduction in the strength of the association between the response and the noncontingent outcome. First, the decrement persisted into extinction when no outcomes were delivered; second, the disruptive effect of the noncontingent outcomes on responding trained with the same outcome was alleviated by signaling those noncontingent outcomes with a Pavlovian conditioned stimulus (CS).

Elements of each of these three demonstrations indicative of outcome encoding in instrumental learning can be found in several studies using Pavlovian condi- 
tioning paradigms. For instance, Cleland and Davey (1982), Holland and Straub (1979), Rescorla (1973), and Stanhope (1989) have found some evidence for a reduction in conditioned responses (CR) following devaluation of the unconditioned stimulus (US). More extensive analyses of the effect of devaluing the first-order stimulus on responding to Pavlovian second-order stimuli have yielded comparable results (Rescorla, 1980). Similarly, observation of the impact of a CS on instrumental performance has frequently served as a probe for assessing the presence of Pavlovian conditioning (Baxter \& Zamble, 1982; Bull, 1970; Bull \& Overmier, 1968; Kruse, Overmier, Konz, \& Rokke, 1983; Rescorla \& LoLordo, 1965). Finally, a number of recent studies have shown that the interposition of USs in a noncontingent fashion undermines the acquisition of Pavlovian conditioning (Durlach, 1983; Durlach \& Shane, 1993; Rescorla, 1984; Stanhope, 1990).

However, close inspection of these studies reveals that few in fact provide solid evidence for a detailed encoding of the features of the US. Many of the studies have employed substantially less complete designs than those available in the instrumental literature. In particular, the use of only one outcome limits the conclusions that may be drawn about the particular features of the outcome that are actually encoded. Without evidence that the manipulations involving a US are specific to CSs associated with that US, one cannot distinguish a detailed encoding of the US from a quite rudimentary encoding that may lack the features needed to differentiate it from other USs. Moreover, some manipulations of the US may affect the subject in a very general way such that the resulting change in behavior would occur even in the absence of any encoding of the US.

Several investigators have noted these criticisms and have employed designs and methodologies analogous to those used to study encoding of the instrumental outcome. Most especially, analyses of pigeon autoshaping procedures have yielded convincing evidence for encoding of the outcome in both first-order (Stanhope, 1989, 1992) and second-order (Colwill \& Rescorla, 1985a; Rescorla, 1980) conditioning. In contrast, these standards have been employed in only a few studies with rats (Kruse et al., 1983). This paper describes our efforts to complement and extend this work to the study of outcome encoding in Pavlovian conditioning using rat subjects.

The purpose of the present study was to employ the kind of experimental designs and techniques used to demonstrate encoding of the outcome in instrumental learning to document further encoding of the outcome in Pavlovian conditioning. In all three studies, rats were exposed to two CSs (one auditory and one visual), each trained as a signal for a different outcome (pellets or sucrose). In Experiment 1, the outcome for one CS was then reduced in value by being paired with a toxin. In Experiment 2, the CSs were presented during a choice extinction test with two instrumental responses trained with those outcomes. In Experiment 3, additional presentations of one of the outcomes were scheduled during the intertrial interval throughout acquisition training. In all three studies, evidence was obtained for a detailed encoding of the outcome as an associate of the Pavlovian CS.

\section{EXPERIMENT 1}

A well-established technique for detecting associations between two events involves changing the value of one event and observing the effect on the other event. The presence of an association may be inferred to the degree that responding to the target event tracks the new value of the altered event. Rescorla and Cunningham (1978) provided an elegant illustration of the use of this logic for detecting associations between elements of a compound stimulus. In their study, rats were given several presentations of two compound stimuli (AX and $\mathrm{BY}$ ). Then $\mathrm{X}$ and $\mathrm{Y}$ were subjected to differential conditioning designed to make $\mathrm{X}$, but not $\mathrm{Y}$, aversive. In a subsequent choice test between $\mathrm{A}$ and $\mathrm{B}$, there was greater rejection of A relative to $\mathrm{B}$. The differential rejection provides unambiguous evidence for the encoding of $\mathrm{X}$ and $\mathrm{Y}$ as associates of $\mathrm{A}$ and $\mathrm{B}$, respectively.

The purpose of the present experiment was to use the outcome devaluation technique to identify encoding of the outcome in Pavlovian conditioning using rat subjects. The design of this study is shown in Figure 1. Rats were given pairings of two stimuli, a tone and a light, each with a different food outcome, pellets or sucrose. Then the value of one of the two outcomes was reduced through pairings with a toxin. In a subsequent extinction test, observations were made of the animals' behavior during nonreinforced presentations of the tone and light CSs. It was anticipated that the frequency of magazinedirected behaviors would be lower during the CS whose outcome had been devalued relative to the CS whose outcome had not been altered.

\section{Method}

Subjects

The subjects were 16 experimentally naive Sprague-Dawley male rats (Holtzman Co.) about 100 days old at the start of the experiment. They were housed in individual cages and maintained at $80 \%$ of their free-feeding weights. Water was available on an adlib schedule.

\section{Apparatus}

The apparatus consisted of four identical Skinner boxes measuring $22.9 \times 20.3 \times 20.3 \mathrm{~cm}$. The two end walls of the chamber were aluminum; the side walls and ceiling were made of Plexiglas.

\begin{tabular}{l|l|l}
\multicolumn{2}{c}{ Design of Experiment 1} \\
Conditioning & Devaluation & Test \\
\hline CS1 $=>01$ & $01+, 02-$ & \multirow{2}{*}{ CS1 v CS2 } \\
\cline { 2 - 2 } $\operatorname{CS} 2=02$ & $02+, 01-$ &
\end{tabular}

Figure 1. Design of Experiment 1: CS1 and CS2 are Pavlovian conditioned stimuli (tone and light); $\mathrm{O} 1$ and $\mathrm{O} 2$ are outcomes (sucrose and pellets); + and - indicate devalued or not devalued. 
Each chamber had a recessed food magazine in the center of one end wall. Pellets were dispensed through a tube onto the floor of the magazine. Sucrose was injected into a small metal cup, measuring $1.25 \mathrm{~cm}$ in diameter and $1.5 \mathrm{~cm}$ deep, located in the floor of each food magazine. A 6-W light located above the food magazine provided constant illumination of the magazine throughout the experiment. The floor of the chamber was composed of 0.48$\mathrm{cm}$ stainless steel rods spaced $1.9 \mathrm{~cm}$ apart. Each Skinner box was enclosed in a sound- and light-resistant shell. Mounted on the inside wall of each shell was a speaker that permitted presentation of an $1800-\mathrm{Hz}$ tone $(\mathrm{T})$ measuring approximately $76 \mathrm{~dB}$ re $20 \mu \mathrm{N} / \mathrm{m}$ and a $6-\mathrm{W}$ light $(\mathrm{L})$. The doors remained open throughout experimental procedures in order to permit video recording. All four boxes and the camera were housed in a soundproof room illuminated by two $40-\mathrm{W}$ bulbs. Experimental events were controlled and recorded automatically by relays and a microprocessor located in an adjoining room. The camera was connected to a video-cassette recorder (VCR) also located in the adjoining room. The VCR was programmed to start recording $15 \mathrm{sec}$ before the onset of each CS and to continue recording until $5 \mathrm{sec}$ after the termination of each trial.

\section{Procedure}

Magazine training. All subjects received one session of magazine training consisting of the delivery of $1045-\mathrm{mg}$ food pellets (Formula A: P. J. Noyes Co.) followed by 10 presentations of $0.3 \mathrm{ml}$ of $8 \%$ sucrose liquid on a variable-time (VT) 60 -sec schedule. In this and all subsequent phases, the delivery of a pellet was always signaled by a distinct click of the pellet dispenser; delivery of sucrose was accompanied by a brief buzzer.

CS preexposure. Following magazine training, the tone $(\mathrm{T})$ and light (L) CSs were preexposed for two sessions. In each session, there were eight 10 -sec presentations of each stimulus with a mean ITI of $120 \mathrm{sec}$. No foods were presented during these sessions.

Pavlovian conditioning. Each session contained 16 conditioning trials on which either T or L was presented for $10 \mathrm{sec}$. All trials terminated with the delivery of either a pellet or sucrose outcome that coincided with the offset of the CS. For half the subjects, T signaled pellets and L signaled sucrose; these combinations were switched for the remaining subjects. Each of the first four sessions contained only one of the two types of stimulus-outcome relationships; two of these sessions contained L conditioning trials, and the other two contained $\mathrm{T}$ conditioning trials. For the remaining 10 sessions, the light CS was presented on 8 trials and the tone $\mathrm{CS}$ on the remaining 8 trials. The order of trial presentations followed a quasi-random sequence that varied across days. The mean ITI was $120 \mathrm{sec}$ throughout conditioning.

US devaluation. An aversion was conditioned to one of the food outcomes in the operant chambers over five 2-day cycles. On Day 1 of each cycle, the to-be-devalued outcome was delivered on a VT 60 -sec schedule for up to 20 min or until five reinforcers had accumulated in the magazine. At the end of the session (or $5 \mathrm{~min}$ after delivery of the last reinforcer), subjects were given a $5-\mathrm{ml} / \mathrm{kg}$ intraperitoneal (i.p.) injection of $0.6 \mathrm{M}$ lithium chloride ( $\mathrm{LiCl}$ ), and were immediately returned to their home cages. On the second day of each cycle, the other outcome was delivered on a VT 60-sec schedule for $20 \mathrm{~min}$. At the end of that session, no injection was administered and the subjects were simply returned to their home cages. Pellets were devalued for half the subjects, and sucrose was devalued for the other half. Designation of the to-be-devalued outcome was balanced across the different stimulus-outcome combinations.

Testing. Two extinction tests were administered. Each test contained eight nonreinforced presentations of each CS with a mean ITI of $120 \mathrm{sec}$. The order of CS presentations followed a repeating sequence of TLLTLTTL. On the day following the second test, two consumption tests were administered to determine the effectiveness of the devaluation treatment. The subjects were placed in the operant chambers, and after $3 \mathrm{~min}$, a reinforcer was delivered. An observer recorded whether or not the reinforcer was consumed within $5 \mathrm{~min}$. Pellet consumption was tested first, and sucrose consumption was tested approximately 30 min later.

Behavioral observation. All observations were made from videotape. Each rat was observed once during the first $5 \mathrm{sec}$ of the CS and once during the second $5 \mathrm{sec}$ of the CS. In addition, two observations of each subject were made during the $10 \mathrm{sec}$ immediately preceding CS onset (pre-CS period). An auditory signal recorded on the videotape was used to pace the observations. The signal sounded every $1.25 \mathrm{sec}$ following the onset of the pre-CS period, and continued throughout the trial. With each pacing signal, the observer depressed one of two keys on a keyboard, depending on the subject's behavior, and then shifted his/her gaze to the next chamber. For each observation, the observer judged whether or not the behavior was a magazine-directed activity. The possible behaviors included in this category were (1) insertion of snout or head into the magazine cavity, (2) standing motionless with all four paws on the ground and with snout within a $10-\mathrm{cm}$ radius of the magazine and pointed toward the magazine, (3) rapid horizontal movements of the head (headjerking) with snout oriented toward the magazine, and (4) approach directly toward the magazine from anywhere in the chamber. In the last three cases, subjects were considered to be oriented toward the magazine if a line extended perpendicularly to the snout tip intersected the magazine. Any behavior that did not fit this description was classified as nonmagazine activity.

Behavioral observations during testing were made independently by two observers, each of whom was blind to the specific conditioning treatments received by the experimental subjects. One of these observers and a different observer also independently scored the final session of conditioning.

\section{Results and Discussion}

The 2 observers scoring the crucial test data agreed on $90 \%$ of their observations. The 2 observers scoring the conditioning data agreed on $88 \%$ of their observations. The data described below come from the observer who scored both the conditioning data and the test data. Statistical analyses of the other observers' data revealed the same pattern of results. A Wilcoxon matched-pairs signedranks test was used to analyze the data reported in this paper. The reported $T$ values are the sum of negative ranks $(T-)$.

Inspection of the observational data from the final conditioning session indicated that the frequency of magazine-directed behavior was higher during the CS periods than during the pre-CS periods. For each subject, the number of observations of magazine-directed behavior was expressed as a percentage of the total observations. The mean percentage of magazine-directed behaviors was 25.4 during the pre-CS period, 70.7 during the first half of the CS, and 89.8 during the second half of the CS. The percentage of magazine observations was significantly higher in both portions of the CS than in the pre-CS period $[T s(16)=0, p<.01]$. In addition, the percentage of magazine behaviors was significantly higher in the second half of the CS than in the first half $[T(14)=0, p<.01]$.

Two points should be noted about these results. First, the ability of the CSs to evoke magazine behaviors is in line with many other reports of the effects of pairing discrete CSs with food USs in rats (Holland, 1977; 1979a, 1979b; Kaye \& Pearce, 1984). Such behaviors have fre- 
quently been used to index successful conditioning. Second, the differential ability of the initial and terminal portions of the CSs to elicit magazine-directed activity is also consistent with previous reports (Holland, 1977). Several explanations for that pattern are possible. Most likely, the lower levels of responding in the first half of the CSs reflect a delay in getting the response initiated. Alternatively, the reduced frequency of conditioned responding in the early component of the CS might reflect the development of inhibition of delay (Pavlov, 1927).

Consumption of the devalued outcome declined over the course of devaluation training. By the fifth cycle, no subject consumed the devalued sucrose and only 2 subjects sampled the devalued pellets. The results of the consumption tests administered after testing indicated that the aversion treatment had been successful. No animal consumed any of the devalued outcomes, but all animals ate the valued outcomes.

Of primary importance are the data from the extinction tests with the two CSs. Figure 2 shows the mean percentage of magazine-directed behaviors observed during the devalued and valued CSs in the first test session. The pair of bars on the left shows the mean observed magazine responses during the first $5 \mathrm{sec}$ of the CS, and the pair of bars on the right shows mean responding during the second $5 \mathrm{sec}$ of the CS. In both cases, magazinedirected behaviors were less likely in the CS trained with the currently devalued outcome than in the CS trained with the valued outcome. This difference was statistically significant for the terminal portion of the CS $[T(15)=15.5, p<.05]$ but not for the initial CS period.

This differential sensitivity to outcome devaluation of stimuli differentially distant from the outcome appears to be a pervasive feature of Pavlovian conditioning. Many studies have found that second-order stimuli may be relatively impervious to changes in the US value that nevertheless have profound effects on responding to

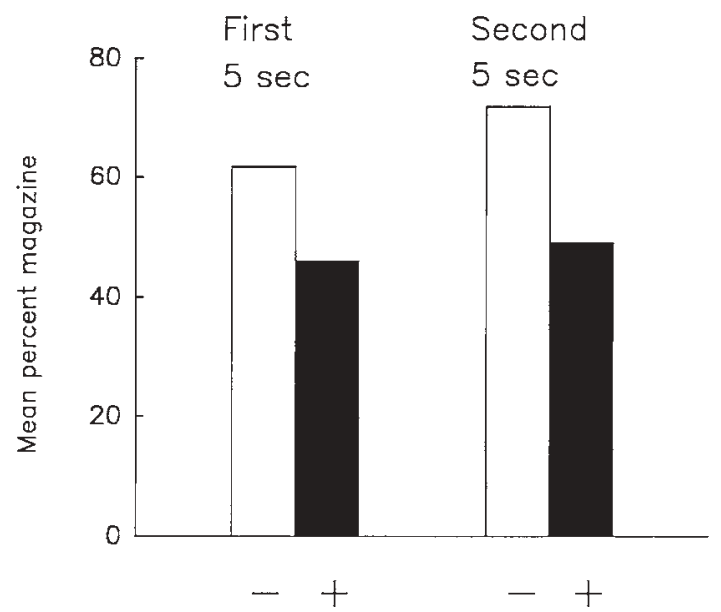

Figure 2. Experiment 1: Mean percentage of magazine-directed behaviors during the first $5 \mathrm{sec}$ (left panel) and second $5 \mathrm{sec}$ (right panel) is shown as a function of whether the CS had been trained with an outcome that was subsequently devalued (filled bar) or not (open bar). first-order stimuli (Holland \& Rescorla, 1975; Stanhope, 1992). Analogous results have been obtained in studies of instrumental learning. For instance, several investigators have found that instrumental behaviors early in the response sequence are depressed less by satiation on the goal than are behaviors late in the sequence leading to the goal (Fantino, 1965; Morgan, 1974; Rescorla, 1977). Similar findings were obtained by Dickinson, Nicholas, and Adams (1983) using a toxin to devalue an outcome; although magazine entries showed some sensitivity to the change in outcome value, leverpresses trained on a variable interval (VI) schedule did not. One explanation frequently given for the temporal gradient underlying sensitivity to outcome revaluation is that only events close to the goal become associated with a detailed representation of that goal.

The observation that outcome devaluation selectively reduced conditioned magazine responses to the terminal portion of the CS paired with that outcome suggests that each CS was associated with a fairly detailed representation of the outcome whose occurrence it had signaled. In the absence of explicit encoding of the distinguishing features of the two outcomes, such differential performance would not have been possible. Although the experimental design contained no explicit control group for assessing the absence of conditioning, it should be recognized that the design arranged for each CS to be paired with one of the USs but, at the same time, to be unpaired with the other US. It is precisely this feature that enabled Rescorla and Cunningham (1978) to exclude nonassociative accounts for the differential rejection of the stimulus whose partner had been devalued. For this reason, the difference in responding to the CSs following outcome devaluation found in Experiment 1 can be attributed with some confidence to the operation of associative processes.

The evidence that devaluation of a US specifically undermines responding to the CS paired with that US is consistent with the results reported by investigators who used rather different outcome devaluation procedures in pigeon autoshaping preparations (Rescorla, 1980; Stanhope, 1989). For example, Stanhope (1989) arranged for one keylight CS to predict water and a different keylight CS to predict food. In a subsequent extinction test, pigeons showed fewer CRs to the CS whose outcome had been devalued through a satiation procedure. Rescorla (1980) found a similar sensitivity in responding to a keylight in a second-order conditioning paradigm following extinction of the first-order keylight with which it had been paired. From these data, it would seem reasonable to infer that learning about the specific features of an outcome occurs across a range of Pavlovian conditioning paradigms.

\section{EXPERIMENT 2}

The purpose of Experiment 2 was to use the transfer technique to investigate encoding of the outcome in Pavlovian conditioning. Of particular relevance to this 
issue are studies by Baxter and Zamble (1982), Kruse et al. (1983), and Colwill and Rescorla (1988). Baxter and Zamble conducted a series of experiments in which they varied the identity of the outcome used to train an instrumental response and a Pavlovian CS. They found that responding was enhanced by presentations of a CS paired with the same outcome (Experiments 1 and 3) but not by presentations of a CS paired with a different outcome (Experiment 2). Kruse et al. examined the influence of a CS on choice of one of two instrumental responses. They found that the CS biased selection of the response that earned the same outcome as that signaled by the CS. Colwill and Rescorla reported modulation of the rate of instrumental responding by a CS. Specifically, a CS depressed performance of an instrumental response trained with a different outcome but left unaffected performance of a response trained with the same outcome. It is notable that, in all these studies, training of the Pavlovian CSs followed training of the instrumental responses in either a free operant (Baxter \& Zamble, 1982) or a discriminated operant procedure (Kruse et al., 1983) or was intermixed with discriminative instrumental training (Colwill \& Rescorla, 1988). It is possible that experience with an instrumental contingency may have affected learning about the Pavlovian CSs and their outcomes. Previous work has shown that discriminative instrumental training affords the opportunity for encoding an outcome as an associate of both the instrumental response and the discriminative stimulus (Colwill \& Rescorla, 1988, 1990). It may have been the case that either or both of these types of learning generalized to learning about the Pavlovian CSs. Thus, the purpose of the present study was to use the transfer procedure to document Pavlovian stimulus-outcome associations in the absence of any influence of instrumental $\mathrm{S}-\mathrm{O}$ or $\mathrm{R}-\mathrm{O}$ associations on the formation of those Pavlovian associations.

Figure 3 shows the design of this experiment. Two stimuli (noise and light) were established as signals for the delivery of two different foods (sucrose and pellets): one stimulus was paired with pellets and the other was paired with sucrose. Following this training, two instrumental responses (leverpress and chain pull) were established, one with pellets and one with sucrose. By conducting this instrumental training after Pavlovian conditioning had already taken place, any encoding of the outcome by the CSs could not be attributed to generalization from other learning. Finally, a choice extinction test with the lever and chain and occasional presentations of the two CSs was administered. The question of interest was whether the CSs would differentially modulate performance of those responses as a function of outcome identity.

\section{Method}

\section{Subjects}

The subjects were 16 experimentally naive Sprague-Dawley male rats (Holtzman Co.) about 100 days old at the start of the experiment. Conditions of housing and maintenance were the same as those described for Experiment 1.

\section{Apparatus}

The apparatus consisted of eight Skinner boxes built to the same specifications as those used in Experiment 1. In addition, each box was equipped with two manipulanda: a lever and a chain. The lever was mounted $9 \mathrm{~cm}$ above the grid floor and $2.5 \mathrm{~cm}$ from the left-hand wall of the food magazine. The chain was suspended from a microswitch mounted on the roof of the chamber. The end of the chain was $11 \mathrm{~cm}$ from the grid floor and $3 \mathrm{~cm}$ from the righthand wall of the food magazine. The same model of microswitch (Unimax Switch Co., 2HBT-1) was used to detect responding on both manipulanda. Access to these manipulanda was prevented by covering the lever with a metal plate and by retracting the chain through an opening in the ceiling. A second speaker mounted on the inside wall of each sound-attenuating shell was used to deliver white noise $(\mathrm{N})$.

\section{Procedure}

All subjects received one session of magazine training in which 10 food pellets (Formula A, P. J. Noyes Co.) followed by 10 sucrose reinforcers $(.3 \mathrm{ml}$ of $8 \%$ sucrose liquid) were delivered on a VT 60 -sec schedule.

Pavlovian conditioning. There were 32 conditioning sessions. Each session contained 16 presentations each of a 30 -sec noise $(\mathrm{N})$ CS and a $30-$ sec light (L) CS with a mean intertrial interval (ITI) of $90 \mathrm{sec}$. Pellets were delivered on a VT $30-\mathrm{sec}$ schedule during one of the stimuli and sucrose was delivered on a VT 30-sec schedule during the other stimulus. For half the subjects, N signaled pellets and L signaled sucrose; these stimulus-outcome combinations were switched for the remaining subjects.

Transfer response training. Leverpressing and chain-pulling were trained initially on a continuous reinforcement (CRF) schedule. Each subject was permitted to earn 25 reinforcers, first with the chain and then with the lever. For half the subjects, leverpressing produced pellets and chain pulling produced sucrose; these response-outcome combinations were switched for the remaining animals. This assignment of response-outcome relations was balanced with respect to the stimulus-outcome relations.

Design of Experiment 2

\begin{tabular}{l|l|l}
$\begin{array}{c}\text { Pavlovian } \\
\text { Conditioning }\end{array}$ & $\begin{array}{c}\text { Transfer } \\
\text { Response Training }\end{array}$ & $\begin{array}{c}\text { Transfer } \\
\text { Test }\end{array}$ \\
\hline CS1 => 01 & R1 => 01 & CS1: R1 V R2 \\
CS2 => 02 & R2 $>02$ & CS2: RI V R2
\end{tabular}

Figure 3. Design of Experiment 2: CS1 and CS2 are Pavlovian conditioned stimuli (noise and light); $\mathrm{O} 1$ and $\mathrm{O} 2$ are outcomes (sucrose and pellets); $\mathrm{R} 1$ and $\mathrm{R} 2$ are instrumental responses (lever and chain). 


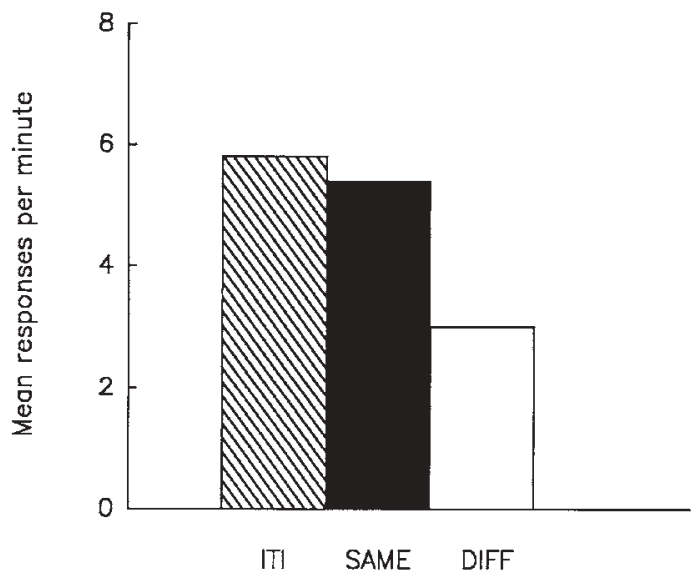

Figure 4. Experiment 2: Mean responses per minute during the first two-trial block of CS presentations. Responding is shown separately when the outcome was the same for both the response and the CS (filled bar) and when it was different (open bar); responding during the ITI is also shown (hatched bar).

Following CRF training, each response was separately reinforced on a VI 30-sec schedule for one 20-min session. In the next four sessions, both responses were available and each was reinforced on a VI 60-sec schedule for $20 \mathrm{~min}$. To encourage sampling of both responses, a reinforcer for one response could not be set up if a reinforcer for the other response had not been collected. Finally, an 8-min extinction test with the lever and chain was administered to all subjects.

Transfer testing. A test session was administered with the lever and chain manipulanda. There were eight presentations of each CS with an ITI of $30 \mathrm{sec}$. No reinforcers were delivered.

\section{Results and Discussion}

All subjects learned to perform the two transfer responses, leverpress and chain pull. The mean rate of responding in the final session of concurrent VI training was 7.5 responses/min. The mean rate of responding in the 8 -min extinction test was 6.8 responses $/ \mathrm{min}$. There was no significant effect of either response or outcome identity in either session.

The results of primary interest are displayed in Figure 4. Responding is shown on the first two test trials with each CS as a function of whether the CS was trained with an outcome that was the same as (filled bar) or different from (blank bar) that earned by the response. There was an immediate differential impact of the CSs on the instrumental responses: there were significantly fewer responses in the CS trained with a different outcome than in the CS trained with the same outcome $[T(14)=6.5, p<.01]$. Moreover, although responding trained with a different outcome was depressed relative to the ITI by a $\operatorname{CS}[T(16)=0, p<.01]$, responding trained with the same outcome did not differ significantly from the ITI. However, responding extinguished very rapidly and these differences disappeared over the course of testing.

The immediate effect of the Pavlovian stimuli on instrumental responding observed in this study replicates the results obtained by Colwill and Rescorla (1988). The present findings suggest that the manner in which Pavlovian stimuli transfer to instrumental responses does not depend on prior instrumental discrimination learning. Furthermore, they reinforce the conclusions of Experiment 1 that the representation of the outcome associated with a Pavlovian CS is quite detailed.

The present findings are also consistent with those from a closely related paradigm introduced by Trapold (1970) to study the nature of the outcome representation in Pavlovian conditioning (see also Carlson \& Wielkiewicz, 1976; Capaldi, Hovancik, \& Friedman, 1976). Briefly, acquisition of discriminative instrumental conditioning is assessed following experience with the instrumental discriminative stimuli in a Pavlovian conditioning procedure. To the degree that changes in the stimulus-outcome relationships between the Pavlovian and the instrumental phases affect instrumental performance, it can be argued that a detailed representation of the outcome is associated with the stimulus in Pavlovian conditioning. An elegant illustration of the use of this transfer of control technique is provided by Trapold (1970). Two stimuli were separately trained as Pavlovian signals for different outcomes; one stimulus was paired with food and one with sucrose. Subjects were then required to learn which of two levers to depress during the two stimuli. For one group, correct responses during a stimulus were followed by the outcome previously paired with that stimulus; for the other group, the outcomes were switched. The problem was solved faster when the stimulus-outcome relationships remained consistent between the two phases, indicating that the subjects must have associated each stimulus with its specific outcome during the Pavlovian conditioning phase.

Design of Experiment 3

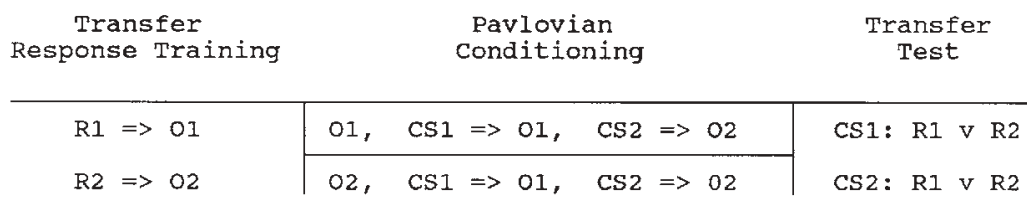

Figure 5. Design of Experiment 3: CS1 and CS2 are Pavlovian conditioned stimuli (noise and light); $\mathrm{O} 1$ and $\mathrm{O} 2$ are outcomes (sucrose and pellets); $\mathrm{R} 1$ and $\mathrm{R} 2$ are instrumental responses (lever and chain). 


\section{EXPERIMENT 3}

The purpose of this study was to assess the effects of unsignaled presentations of an outcome on the development of stimulus-outcome associations. As mentioned previously, several investigators have found that the formation of a stimulus-outcome association may be disrupted by the insertion of unsignaled presentations of the outcome in the intertrial interval. Analysis of this effect has suggested that the formation of the stimulusoutcome association is blocked by the association that develops between the context and that outcome (Durlach, 1983; Rescorla, 1984). To the degree that detailed representations of the outcome become associated with Pavlovian stimuli, it would be anticipated that stimulusoutcome learning would be most severely disrupted by the presentation of identical ITI outcomes.

Figure 5 illustrates the design of this experiment which was modeled after that used by Colwill and Rescorla (1986) to study the effects of the identity of ITI outcomes on instrumental learning. Rats were exposed to presentations of two different stimuli, one signaling pellets and one signaling sucrose. Deliveries of one of those outcomes were also scheduled during the ITI at a rate equal to that in effect during presentation of the stimuli. In this way, the ITI outcome was the same as the outcome following one of the CSs (random CS) but different from the outcome following the other CS (positive $\mathrm{CS}$ ). Following this training, both stimuli were tested for their conditioning. This was accomplished by examining the effect of their presentation on performance of two instrumental responses, one of which had earned pellets and the other of which had earned sucrose. The question of interest concerned the ability of the two stimuli to modulate those responses. It was expected that the random CS would have little, if any, differential effect on those responses, but that in the presence of the positive $\mathrm{CS}$, responses trained with the outcome predicted by that CS would be more likely than responses trained with a different outcome.

Method
Subjects
Thirty-one experimentally naive male Holtzman-derived
Sprague-Dawley rats (Harlan Co.) served as subjects. They were
housed and maintained in the same way as animals in the two pre-
vious experiments.
Apparatus
The apparatus consisted of eight Skinner boxes built according
to the same specifications as those used in Experiment 2 with two
exceptions. First, the sucrose reinforcer was delivered through a
tube inserted through the roof of the food magazine. Each opera-
tion of the sucrose dispenser allowed 0.2 ml of $8 \%$ sucrose to flow
onto the floor of the food magazine, where it collected in a shal-
low indentation. Second, the lever was mounted 2.5 cm from the
right-hand wall of the food magazine, and the chain was sus-
pended 3 cm from the left-hand wall of the food magazine. Fur-
thermore, four of the boxes contained a retractable lever which
was withdrawn in order to prevent access to it. Experimental
events were controlled and recorded automatically by interfacing

(Med Associates) and an XT microprocessor located in an adjoining room.

\section{Procedure}

All subjects received one session of magazine training consisting of the delivery of ten 45-mg food pellets (Formula A: P. J. Noyes Co.), followed by 10 presentations of $0.2 \mathrm{ml}$ of $8 \%$ sucrose liquid on a VT 60 -sec schedule. In this and all subsequent phases, a brief click accompanied operation of the pellet and sucrose dispensers. During this phase, the response manipulanda were inaccessible to the animals.

Transfer-response training. The transfer responses were trained prior to conditioning in order to minimize the impact that differential associations of the two outcomes with the background might have on learning the response-outcome associations. Lever and chain were reinforced, one with pellets and one with sucrose. Training was identical to that described for Experiment 2.

Pavlovian conditioning. Following training of the transfer responses, subjects were given 32 conditioning sessions. Each session contained eight presentations of a 30 -sec light CS intermixed with eight presentations of a $30-\mathrm{sec}$ noise CS. The mean ITI was $90 \mathrm{sec}$. During each CS, reinforcers were delivered on a VT 30-sec schedule. For half the animals, pellets were presented during $\mathrm{L}$ and sucrose during $\mathrm{N}$; these stimulus-outcome combinations were switched for the remaining animals. During the ITI, one of the reinforcers was presented on a VT 30-sec schedule; for half the animals, the ITI reinforcer was pellets and for the others it was sucrose. Thus, each animal was trained with two CSs, one of which signaled an outcome that was the same as that presented during the ITI (random CS) and the other of which signaled an outcome different from that occurring during the ITI (positive CS). For purposes of exposition, it is convenient to refer to the stimulus trained with the outcome that was also delivered during the ITI as a random CS. However, depending upon the inclusion or exclusion of the duration of the positive CS in the calculations, the actual contingency between the random CS and its outcome may have been slightly positive rather than random.

Transfer testing. Four extinction tests were administered, each of which contained eight nonreinforced presentations each of the noise and light CSs. The ITI was $30 \mathrm{sec}$. The lever and chain were simultaneously available in these test sessions, but responding was never reinforced. The first two tests were conducted after 16 sessions of Pavlovian conditioning; the remaining two test sessions were administered after an additional 16 sessions of Pavlovian conditioning. Prior to each of the two pairs of test sessions, all subjects received two additional 20 -min sessions of concurrent VI training with the lever and the chain.

\section{Results and Discussion}

As noted previously, $T$ values are the sum of negative (least frequent) ranks; $z$ scores were calculated using the method suggested by Tate and Clelland (1957).

Initial training of leverpressing and chain-pulling proceeded smoothly with all but 2 animals. Both subjects failed to chain-pull reliably for sucrose and their data were not included in subsequent analyses. Analysis of the VI training session immediately preceding the start of Pavlovian conditioning found no significant difference between rates of responding for sucrose or pellets ( 9.3 and 9.4 responses/min, respectively). However, a comparison of leverpressing and chain-pulling revealed significantly higher rates of leverpressing than of chainpulling [10.4 and 8.3 responses/min, respectively; $T(29)=111.5, z=-2.28, p<.05]$. This difference between the two responses persisted throughout subsequent 


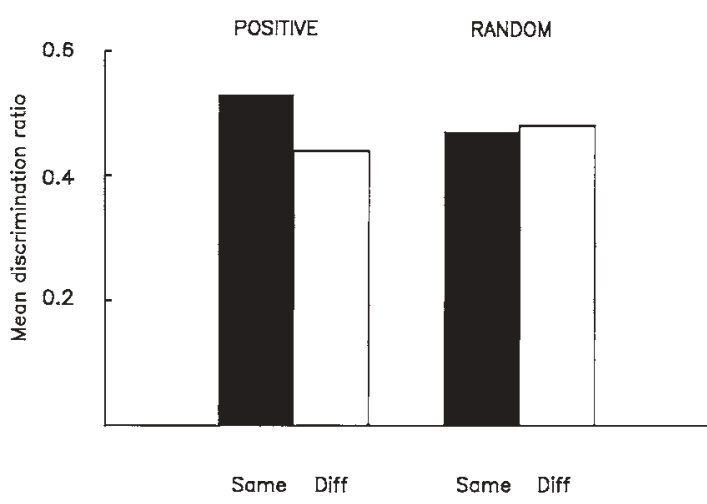

Figure 6. Experiment 3: Mean discrimination ratios for the positive (left panel) and random (right panel) CSs in the second test series. Data are shown separately when the response and stimulus share the same outcome (filled bar) and when they have different outcomes (open bar).

sessions of VI training. Inspection of each VI training session preceding each of the two test series found higher rates of leverpressing than of chain-pulling [7.7 vs. 6.1 and 9.3 vs. 7.6 responses $/ \mathrm{min} ; T \mathrm{~s}(29)=94.5$, $z \mathrm{~s}=-2.64, p \mathrm{~s}<.01]$. For this reason, individual discrimination ratios were calculated in each test session so that any differential impact of a CS on the two responses would not be obscured by this baseline difference. Ratios were derived for each animal by dividing the response rate during the CS by the sum of that rate and the response rate during the ITI.

The data of most interest concern the impact of the two CSs on instrumental responses trained with either the same or different outcomes. The data from the first test series were encouraging. Responding in the presence of the positive CS was significantly higher for the same outcome than for the different outcome $[.53$ and .48 , respectively; $T(28)=115.5, z=-1.98, p<.05]$. Although the effect of the random CS was similar in direction to that produced by the positive $\mathrm{CS}$, the difference between the same and different discrimination ratios was not significant [.50 and .46, respectively; $T(28)=133.5, z=-1.57, p>.10]$.

Figure 6 shows the data from the second test series. The pair of bars on the left show mean discrimination ratios for the CS trained with different ITI outcomes, and the pair of bars on the right show the data for the CS trained with same ITI outcomes. In each case, mean discrimination ratios are shown separately as a function of the identity relationship between the outcome for the CS and the outcome for the response. Two aspects of these data should be noted. First, the positive CS continued to exert an outcome-specific effect on instrumental responding. The mean discrimination ratio for different responses was significantly lower than that for same responses [.44 and .53, respectively; $T(29)=89.5, z=$ $-2.75, p<.01]$. This result replicates that of Experiment 2. Second, the random CS did not differentially modulate the instrumental responses as a function of outcome identity. There was no significant difference between same and different responses [.47 and .48, respectively; $T(29)=206, z=-0.23, p>.10$.

The pattern of these results suggests that the insertion of intertrial USs selectively undermines learning about stimuli that are paired with that same outcome. Inspection of the response rates during the intertrial intervals in the critical test sessions provides some support for the view that the source of that interference involves contextUS associations. To the degree that the context becomes a signal for the outcome presented during the ITI, instrumental responses trained with that ITI outcome should be more likely than responses trained with a different outcome. Although analysis of ITI responding in the first series of test sessions found no significant difference between performance of the response trained with the ITI outcome (3.9 responses/min) and the response trained with a different outcome (4.1 responses/ min), analysis of the second test series yielded a small effect. During the ITI, the rate of responding trained with the ITI outcome was slightly, but significantly, higher than that trained with a different outcome [3.0 vs. 2.7 responses $/ \mathrm{min}$, respectively, $T(28)=107.5, z=$ $-2.16, p<.05]$. Thus, the contextual cues exerted an outcome-specific effect on performance of the instrumental responses similar to that found with discrete Pavlovian CSs.

In summary, these results provide additional support for the view that Pavlovian conditioning involves associations between stimuli and fairly detailed representations of the outcomes whose occurrence they signal. Moreover, they confirm the results recently obtained by Stanhope (1992) in her studies of outcome encoding in the pigeon autoshaping preparation.

\section{GENERAL DISCUSSION}

Three techniques that have been used to probe the content of outcome representations in instrumental learning were employed in the present investigations of Pavlovian conditioning. Using outcome devaluation, we found, with Experiment 1, that a CS whose outcome had been devalued elicited fewer magazine-directed responses than a CS whose outcome was not altered. Using transfer, we found, with Experiment 2, that a CS selectively depressed performance of an instrumental response trained with a different outcome but had no effect on performance of a response trained with the same outcome. Using sensitivity to CS-US contingency, we found, with Experiment 3, that noncontingent outcomes interfered with development of associations between a CS paired with that outcome but not with a CS paired with a different outcome. The dependence of the effects of each of these manipulations on outcome identity provides support for a detailed encoding of the outcome in Pavlovian conditioning.

The evidence presented here for learning about the identity of the outcome in Pavlovian conditioning confirms conclusions drawn from three other sources. First, 
a number of studies have shown that the topography of the CR depends upon the identity of the US (Davey \& Cleland, 1982; Hawkins, Lalevic, Clark, \& Kandel, 1989; Jenkins \& Moore, 1973; LaMon \& Zeigler, 1984; Skelton, Spetch, \& Wilkie, 1980; Spetch, Wilkie, \& Skelton, 1981; Walters, 1989). In a classic study of autoshaping, Jenkins and Moore (1973) observed that the response to the CS captured features characteristic of the response to the US paired with that CS. Specifically, pigeons pecked a keylight CS that signaled grain as if they were eating, and pecked a keylight CS that signaled water as if they were drinking. These kinds of observations of the influence of the outcome on the nature of the $\mathrm{CR}$ are consistent with the claim that the CS activates a fairly specific representation of its outcome. However, it is of some importance to note the superiority of the present battery of techniques for identifying encoding of the outcome to the traditional method of comparing CR and UR topographies. That method is, of course, restricted to those instances where the USs support different behaviors. In contrast, the techniques employed in the present studies may also be used to analyze encoding of outcomes that support identical patterns of behavior. For instance, in Experiment 1, two different USs (pellets and sucrose liquid) supported the same CR (magazine-directed activity). Casual observation of that response revealed no obvious basis for discerning whether the CS signaled pellets or sucrose. Yet, changing the value of the outcome differentially affected that $\mathrm{CR}$, indicating that the representations of the two outcomes were, in fact, quite distinct.

Second, Pavlovian conditioning has been shown to be sensitive to variations in the consistency of the relationship between a stimulus and its outcome. For example, Rescorla (1980) found second-order conditioning to a stimulus paired with the same outcome across trials to be superior to the conditioning of a stimulus whose outcome differed across trials. The fact that changes in the sensory features of the outcome interfered with acquisition of second-order conditioning encourages the view that those features participated in the outcome representation that was associated with the second-order CS.

Third, the results of the present studies are consistent with those from several studies that used outcome manipulations in a conventional blocking paradigm. Several authors have reported that conditioning of a stimulus will not be reduced or blocked by the presence of a pretrained CS if the outcome used for conditioning is altered in either quantity (Dickinson \& Mackintosh, 1979; Holland, 1984) or quality (Holland, 1988; Stickney \& Donahoe, 1983) from that used for pretraining. However, such outcome manipulations do not inevitably produce unblocking (e.g., Bakal, Johnson, \& Rescorla, 1974; Ganesan \& Pearce, 1988). The reasons for these discrepancies are not entirely clear, but comparisons between those procedures and the present ones suggest that the manner in which outcome specificity is detected may be crucial. While our studies employed within-subject designs, unblocking experiments have routinely em- ployed between-subject designs. The relative insensitivity of between-subject comparisons to small differences has been discussed elsewhere (e.g., Colwill \& Rescorla, 1985b; Rescorla \& Cunningham, 1978).

The strength of the present findings lies in their ability to reveal that learning about the distinctive features of an outcome occurs in Pavlovian conditioning. However, it is noticeable that the effects indicative of outcome specificity were all quite modest in size and disappeared rapidly during testing. One possibility suggested by this is that features common to the different outcomes may have also been associated with the CSs. Although the two food outcomes used in the present experiments differed in their sensory attributes (for example, texture and flavor), they shared a positive affective component. The fact that outcome devaluation in Experiment 1 did not eliminate entirely the ability of the CS trained with that outcome to evoke its $\mathrm{CR}$ during testing is consistent with learning about the affective aspect of the outcome (Mackintosh, 1983; Rescorla, 1980). Moreover, depending upon the relative strengths of the connections of the CS with the unique sensory and shared affective features, the magnitude of the residual behavior might be quite considerable. A relatively weaker association with the unique features than with the common features of the outcome would also account for the rapid loss of outcome-specific transfer in Experiment 2. Although progress has been made on the identification of factors that influence the relative contributions of associations with the sensory and affective components of an outcome in Pavlovian second-order conditioning (Rescorla, 1980), it has yet to be determined whether those manipulations influence outcome encoding in first-order conditioning.

The present results reinforce the parallels that have been drawn between instrumental response-outcome associations and Pavlovian stimulus-outcome associations (Colwill \& Rescorla, 1986; Mackintosh, 1983). But in so doing, they prompt reconsideration of the role Pavlovian conditioning processes might play in mediating the outcome-dependent effects observed in studies of instrumental learning. For instance, it might be argued that the depressive effect of outcome devaluation on an instrumental response is mediated by withdrawal from response-correlated stimuli that serve as Pavlovian signals for the devalued outcome. Colwill and Rescorla (1986) noted that this argument is difficult to counter empirically. However, they reported an experiment in which they successfully recorded an outcome devaluation effect under conditions that minimized any differences in the stimulus events accompanying performance of the instrumental responses (deflections of a joystick manipulandum). Although interpretation of their results in terms of stimulus correlates of response execution is not impossible, it would seem to render the distinction between responses and stimuli inconsequential, at least in terms of any theoretical significance (see Colwill, 1994, for further discussion of this issue).

The conclusions about outcome encoding that emerge from the present studies are not novel. Indeed, as we 
have previously acknowledged, evidence based on the use of the present techniques for identifying at least a rudimentary encoding of the outcome may be found in several other reports. However, one of the virtues of the experiments reported in this paper is that, by using more sophisticated designs, they provide unambiguous evidence for the view that Pavlovian conditioning may involve associations between a stimulus and a detailed representation of the outcome that is contingent upon that stimulus.

\section{REFERENCES}

Bakal, C. W., Johnson, R. D., \& Rescorla, R. A. (1974). The effect of change in US quality on the blocking effect. Pavlovian Journal of Biological Sciences, 9, 97-103.

Baxter, D. J., \& Zamble, E. (1982). Reinforcer and response specificity in appetitive transfer of control. Animal Learning \& Behavior, 10, 201-210.

Bull, J. A., III (1970). An interaction between appetitive Pavlovian CSs and instrumental avoidance responding. Learning \& Motivation, 1, 18-26.

Bull, J. A., III, \& Overmier, J. B. (1968). Additive and subtractive properties of excitation and inhibition. Journal of Comparative \& Physiological Psychology, 66, 511-514.

Capaldi, E. D., HovanciK, J. R., \& Friedman, F. (1976). Effects of expectancies of different reward magnitudes in transfer from noncontingent pairings to instrumental performance. Learning \& $\mathrm{Mo}$ tivation, 7, 197-210.

Carlson, J. G., \& Wielkiewicz, R. M. (1976). Mediators of the effects of magnitude of reinforcement. Learning \& Motivation, 7 , 184-196.

Cleland, G. G., \& Davey, G. C. L. (1982). The effects of satiation and reinforcer devaluation on signal-centered behavior in the rat. Learning \& Motivation, 13, 343-360.

Colwill, R. M. (1994). Associative representations of instrumenta contingencies. In D. L. Medin (Ed.), The psychology of learning and motivation (Vol. 28, pp. 1-72). New York: Academic Press.

Colwill, R. M., \& Rescorla, R. A. (1985a). Extensive training, partial reinforcement, and temporal gaps do not affect S-S learning in second-order autoshaping. Animal Learning \& Behavior, 13, 165170

Colwill, R. M., \& Rescorla, R. A. (1985b). Post-conditioning devaluation of a reinforcer affects instrumental responding. Journal of Experimental Psychology: Animal Behavior Processes, 11, 120132

Colwill, R. M., \& Rescorla, R. A. (1986). Associative structures in instrumental learning. In G. H. Bower (Ed.), The psychology of learning and motivation (Vol. 20, pp. 55-104). New York: Academic Press.

Colwill, R. M., \& Rescorla, R. A. (1988). Associations between the discriminative stimulus and the reinforcer in instrumental learning. Journal of Experimental Psychology: Animal Behavior Processes, 14, 155-164.

Colwill, R. M., \& Rescorla, R. A. (1990). Effect of reinforcer devaluation on discriminative control of instrumental behavior. Journal of Experimental Psychology: Animal Behavior Processes, 16, 40-47.

Davey, G. C. L., \& Cleland, G. G. (1982). Topography of signal-centered behavior in the rat: Effects of deprivation state and reinforcer type. Journal of the Experimental Analysis of Behavior, 38, 291304

Dickinson, A., \& MACKInTOSH, N. J. (1979). Reinforcer specificity in the enhancement of conditioning by posttrial surprise. Journal of Experimental Psychology: Animal Behavior Processes, 5, 162-177.

Dickinson, A., Nicholas, D. J., \& Adams, C. D. (1983). The effect of the instrumental training contingency on susceptibility to reinforcer devaluation. Quarterly Journal of Experimental Psychology, 35B, 35-51.
DURLACH, P. J. (1983). The effect of signalling intertrial USs in autoshaping. Journal of Experimental Psychology: Animal Behavior Processes, 9, 374-389.

Durlach, P. J., \& Shane, D. O. (1993). The effect of intertrial food presentations on anticipatory goal-tracking in the rat. Quarterly Journal of Experimental Psychology, 46B, 289-318.

Fantino, E. (1965). Some data on the discriminative stimulus hypothesis of secondary reinforcement. Psychological Record, $\mathbf{1 5}$ 409-415.

Ganesan, R., \& Pearce, J. M. (1988). Effect of changing the unconditioned stimulus on appetitive blocking. Journal of Experimental Psychology: Animal Behavior Processes, 14, 280-291.

Hawkins, R. D., Lalevic, N., Clark, G. A., \& Kandel, E. R. (1989) Classical conditioning of the aplysia siphon-withdrawal reflex exhibits response specificity. Proceedings of the National Academy of Sciences, 86, 7620-7624.

Holland, P. C. (1977). Conditioned stimulus as a determinant of the form of the Pavlovian conditioned response. Journal of Experimental Psychology: Animal Behavior Processes, 3, 77-104.

Holland, P. C. (1979a). Differential effects of omission contingencies on various components of Pavlovian appetitive conditioned responding in rats. Journal of Experimental Psychology: Animal Behavior Processes, 5, 178-193.

Holland, P. C. (1979b). The effects of qualitative and quantitative variation in the US on individual components of Pavlovian appetitive conditioned behavior in rats. Animal Learning \& Behavior, 7, 424-432.

Holland, P. C. (1984). Unblocking in Pavlovian appetitive conditioning. Journal of Experimental Psychology: Animal Behavior Processes, 10, 476-497.

Holland, P. C. (1988). Excitation and inhibition in unblocking. Journal of Experimental Psychology: Animal Behavior Processes, 14 261-279.

Holland, P. C., \& Rescorla, R. A. (1975). Second-order conditioning with food unconditioned stimulus. Journal of Comparative \& Physiological Psychology, 88, 459-467.

Holland, P. C., \& Straub, J. J. (1979). Differential effects of two ways of devaluing the unconditioned stimulus after Pavlovian appetitive conditioning. Journal of Experimental Psychology: Animal Behavior Processes, 5, 65-78.

Jenkins, H. M., \& Moore, B. R. (1973). The form of the autoshaped response with food or water reinforcers. Journal of the Experimental Analysis of Behavior, 20, 163-181.

Kaye, H., \& Pearce, J. M. (1984). The strength of the orienting response during Pavlovian conditioning. Journal of Experimental Psychology: Animal Behavior Processes, 10, 90-109.

Kruse, J. M., Overmier, J. B., Konz, W. A., \& Rokke, E. (1983). Pavlovian conditioned stimulus effects upon instrumental choice behavior are reinforcer specific. Learning \& Motivation, 14, 165 181.

LaMon, B. C., \& Ziegler, H. P. (1984). Grasping in the pigeon (Columbia livia): Stimulus control during conditioned and consummatory responses. Animal Learning \& Behavior, 12, 223-231.

Mackintosh, N. J. (1983). Conditioning and associative learning. Oxford: Oxford University Press.

Morgan, M. J. (1974). Resistance to satiation. Animal Behaviour, 22, 449-466.

Pavlov, I. P. (1927). Conditioned reflexes (G. V. Anrep, Trans.). London: Oxford University Press.

Rescorla, R. A. (1973). Effect of US habituation following conditioning. Journal of Comparative \& Physiological Psychology, 82 137-143.

Rescorla, R. A. (1977). Pavlovian second-order conditioning: Some implications for instrumental behavior. In H. Davis \& H. M. B. Hurwitz (Eds.), Operant-Pavlovian interactions (pp. 133-164). Hillsdale, NJ: Erlbaum.

Rescorla, R. A. (1980). Pavlovian second-order conditioning. Hillsdale, NJ: Erlbaum

Rescorla, R. A. (1984). Signaling intertrial shocks attenuates their negative effect on conditioned suppression. Bulletin of the Psychonomic Society, 22, 225-228.

Rescorla, R. A., \& Cunningham, C. L. (1978). Within-compound 
flavor associations. Journal of Experimental Psychology: Animal Behavior Processes, 4, 267-275.

Rescorla, R. A., \& LoLordo, V. M. (1965). Inhibition of avoidance behavior. Journal of Comparative \& Physiological Psychology, 59, 406-412.

Skelton, R. W., Spetch, M. L., \& Wilkie, D. M. (1980). A method for automatically recording topographical differences in pigeons' keypecking for food and water reinforcers. Behavior Research Methods \& Instrumentation, 12, 349-352.

Spetch, M. L., Wilkie, D. M., \& Skelton, R. W. (1981). Control of pigeons' keypecking topography by a schedule of alternating food and water reward. Animal Learning \& Behavior, 9, 223-229.

Stanhope, K. J. (1989). Dissociation of the effect of reinforcer type and response strength on the force of a conditioned response. Animal Learning \& Behavior, 17, 311-321.

Stanhope, K. J. (1990). Effect of intertrial unconditioned stimulus (US) presentations upon responding to a conditioned stimulus predictive of either the same or a different appetitive US. Learning \& Motivation, 21, 266-286.
Stanhope, K. J. (1992). The representation of the reinforcer and the force of the pigeon's keypeck in first- and second-order conditioning. Quarterly Journal of Experimental Psychology, 44B, 137-158.

STICKNEY, K. J., \& Donahoe, J. W. (1983). Attenuation of blocking by a change in US locus. Animal Learning \& Behavior, 11, 60-66.

Tate, M. W., \& Clelland, R. C. (1957). Nonparametric and shortcut statistics in the social, biological and medical sciences. Danville, IL: Interstate Printers and Publishers.

Trapold, M. A. (1970). Are expectancies based upon different positive reinforcing events discriminably different? Learning \& Motivation, 1, 129-140.

Walters, E. T. (1989). Transformation of siphon responses during conditioning of aplysia suggests a model of primitive stimulusresponse association. Proceedings of the National Academy of Sciences, 86, 7616-7619.

(Manuscript received December 6, 1991; revision accepted for publication April 1, 1994.) 